\title{
Selective synthesis of polyamines in multipurpose plants.
}

\author{
Francesco Maestria ${ }^{\text {, Federico Sacco }}{ }^{b}$, Carlo Pirola ${ }^{b}$, Renato Rota $^{a, *}$ \\ a Politecnico di Milano, Dipartimento di Chimica, Materiali e Ingegneria Chimica “G. Natta”, \\ Piazza Leonardo da Vinci, 32 - 20133 Milano - Italy \\ ${ }^{\mathrm{b}}$ Università degli Studi di Milano, Dipartimento di Chimica, \\ via Golgi, 19 - 20122 Milano - Italy \\ fax: +39 0223993180 ; e-mail: *renato.rota@polimi.it
}

\begin{abstract}
$\underline{\text { Abstract }}$
A number of reaction processes undergo yield drops since an intermediate target species can further react with one of the reactants generating lower volatility by-products. Such reactions are typically fast and non-selective, so that a gradual conversion of the reactants is recommended because of safety and quality constraints; when dealing with relatively low-volumes of a highly fragmented set of products, a semibatch reactor is normally adopted where the reaction selectivity is increased by increasing the excess of the selective reactant, therefore lowering the plant productivity.

For increasing the process selectivity without reducing the plant productivity a different reactor configuration has been recently proposed: the so-called semibatch recycle reactor. However, the good performance of this configuration has never been experimentally confirmed.

In this work, the unselective industrial synthesis of polyamines through alkylation of 1,2dichloroethane with a diamine has been experimentally investigated both in a standard semibatch reactor as well as in a semibatch recycle reactor, showing that the latter can lead to a significant increase of both the process selectivity and productivity.
\end{abstract}

Keywords: Multipurpose processes; Selectivity; Safety; Semibatch Reactor (SBR); Semibatch Recycle Reactor (SBRR); Polyamines. 


\section{Introduction}

In the fine chemical as well as in the intermediates chemical industry a wide range of products of the same class are often manufactured on a campaign basis, so that multipurpose batch or semibatch reactors are adopted instead of continuous ones (Dimian et al., 2014).

In a number of such processes the target reaction product can unselectively react with one of the reactants, generating undesired by-products (Westerterp et al., 1984), which must be then separated from the target one and finally disposed as a waste.

A number of such reactions can be found even in the intermediates chemical industry (Arpe, 2010;

Garner and Nunes, 1973): as a relevant example the production of polyamines from 1,2dichloroethane (DCE) and diamines is discussed in this work.

Polyamines have many industrial uses, ranging from the curing of epoxy resins, to the production of functionalized polyamides and chelating agents (Garner and Nunes, 1973). Moreover, polyamines are a valuable backbone for the synthesis of a number of fine chemical additives.

Starting from several diamines, the range of polyamines which can be generated for specialized applications is relatively wide, so that they are often produced in non-continuous multipurpose SBRs, in which DCE (that is, the non-selective reactant) is gradually added to a diamine excess, to control both the reaction heat and the potential gas or vapor evolution (Steinbach, 1999; Maestri and Rota, 2016; Copelli et al., 2010).

The selectivity of such processes is often limited since DCE can further react with the target bisaminoalkyl ethylenediamine to generate heavier derivatives, which affect the process yield and the purity of the final product when they are not separated from it.

In such cases, when performing the reaction in a standard SBR, a huge excess of the diamine must be adopted in order to keep the process selectivity at acceptable values: however, such process conditions lower the plant productivity, also because of the subsequent separation of the diamine excess. 
Moreover, in some cases such a separation is cumbersome through conventional distillation because of both a relatively low volatility difference between the components to be separated, and a potential azeotropic behavior of the mixture (Hiraga et al., 1986). This calls for more sophisticated separation techniques, with a significant increase of the production costs.

Whereas for some applications the raw polyamine can be used without relevant drawbacks, there are systems in which a higher purity of the product is required: for the sake of example, when high molecular weight polyamides must be generated through reaction of the polyamine with a bifunctional acid, the stoichiometric ratio between the two reactants is a key parameter, which can be guaranteed only starting from relatively pure species (Garner and Nunes, 1973).

To overcome the selectivity problem or when the required diamine is difficult to be found on the market, alternative synthesis routes are adopted: for the sake of example, N,N'-bis-(3aminopropyl)-ethylenediamine is industrially produced through Michael addition of acrylonitrile to ethylenediamine, followed by catalytic hydrogenation of the intermediate bis-cyanoethyl derivative (Häussling et al., 1998). However, the process involves two reaction steps, one of which (that is, the hydrogenation) requires a technology not always readily available.

When producing polyamines through direct alkylation of DCE with a relatively volatile diamine, the process selectivity and productivity can be increased even in a non-continuous reaction system adopting the semi batch recycle reactor (SBRR) process (Maestri and Rota, 2013 and 2015). In such a plant configuration the diamine is refluxed through the system and the DCE dosing is spread over a suitable time period, in order to minimize the target product accumulation in the reactor. The resulting segregation between the target product and the non-selective reactant allows for effectively limiting the extent of the further alkylation of the target polyamine by DCE and hence for achieving a high process selectivity, even adopting a limited initial excess of the starting diamine.

In this work, the selectivity behavior of the reaction between 1,2-dichloroethane (DCE) and ethylenediamine (EDA) to produce triethylenetetramine (TETA) has been experimentally tested in a 
standard SBR and in a SBRR, in which DCE is dosed in refluxing EDA.

It has been experimentally demonstrated (for the first time, in the Author's knowledge) that through the SBRR process configuration a relevant increase of the reaction selectivity can be achieved, even dealing with a reaction system in which more than one side reaction can occur: this allows for reaching a purity of the product mixture much higher than that of the product commonly available on the market (Akzo Nobel, 2018; Huntsman, 2018).

\section{SBRR process configuration}

When dealing with a semibatch reaction process of the type:

$\left\{\begin{array}{l}A+B \stackrel{k_{1}}{\rightarrow} C \\ A+C \stackrel{k_{2}}{\rightarrow} D\end{array}\right.$

according to which a low volatility target product $\mathrm{C}$ can further react with a dosed unselective reactant $A$ to generate a by-product $\mathrm{D}$, the semibatch recycle reactor (SBRR) (Maestri and Rota, 2013 and 2015) can be used to achieve both a high process selectivity and productivity.

In such a plant configuration the selective reactant B is refluxed at a given rate between a bottom reboiler and a top reaction vessel, after separating the target product $\mathrm{C}$ from a volatile reaction mixture, mainly consisting of unreacted B (see Figure 1). The coreactant A is fed to the top reactor at a relatively low flowrate, so as to minimize the accumulation of $\mathrm{C}$ in the reactor. In this way, the target product $\mathrm{C}$ can be effectively removed from the reactor and is therefore protected from its further degradation, even operating the reactor at a relatively low initial excess of the selective reactant B.

The SBRR process configuration allows therefore for operating the reactor continuously even within a non-continuous system. Moreover, as a relevant advantage with respect to a continuous reactor, the average residence time in the reaction vessel can be adjusted in a much more flexible way and the lower excess of the selective reactant for a given process selectivity allows for increasing the plant productivity. 
Finally, the reaction heat can be effectively removed through the continuous reflux of the unconverted reaction mass, performing at the same time the reaction at a lower temperature than the normal boiling point of $\mathrm{B}$ : this provides a relevant degree of flexibility with respect to a standard reflux SBR, which in such a case should be operated under vacuum.

For a SBRR system with a power law kinetics, the time evolution of the B reactant conversion:

$\chi=\frac{\mathrm{n}_{\mathrm{B} 0}-\mathrm{n}_{\mathrm{B}}}{\mathrm{n}_{\mathrm{A} 1}}$

of the B reactant selectivity to product C:

$\sigma=\frac{\mathrm{n}_{\mathrm{C}}}{\mathrm{n}_{\mathrm{B} 0}-\mathrm{n}_{\mathrm{B}}}$

and of the dimensionless concentration of product $C$ in the top reactor, $\gamma_{C, r}$ :

$\gamma_{\mathrm{C}, \mathrm{r}}=\frac{\mathrm{C}_{\mathrm{C}, \mathrm{r}}}{\mathrm{C}_{\mathrm{B}, \mathrm{r}}}$

is described by the following ODEs system (Maestri and Rota, 2013 and 2015):

$\frac{\mathrm{d} \chi}{\mathrm{d} \vartheta}=\operatorname{Da}_{1} \frac{1}{\mathrm{Ex}^{\mathrm{n}-1}}\left[\vartheta-\chi\left(2-\sigma_{\mathrm{C}}\right)\right]^{\mathrm{n}}\left(1-\frac{\chi}{\mathrm{Ex}}\right)^{\mathrm{m}} \kappa_{1}$

$\frac{\mathrm{d} \sigma_{\mathrm{C}}}{\mathrm{d} \vartheta}=\operatorname{Da}_{1} \frac{1}{\mathrm{Ex}^{\mathrm{n}-1}} \frac{1}{\chi}\left[\vartheta-\chi\left(2-\sigma_{\mathrm{C}}\right)\right]^{\mathrm{n}}\left(1-\frac{\chi}{\mathrm{Ex}}\right)^{\mathrm{m}} \kappa_{1}+$

$-\mathrm{Da}_{2} \frac{1}{\mathrm{Ex}^{\mathrm{p}-1}} \frac{1}{\chi}\left[\vartheta-\chi\left(2-\sigma_{\mathrm{C}}\right)\right]^{\mathrm{p}} \gamma_{\mathrm{C}, \mathrm{r}} \mathrm{q}_{\kappa_{2}}-\frac{\sigma_{\mathrm{C}}}{\chi} \frac{\mathrm{d} \chi}{\mathrm{d} \vartheta}$

$\frac{\mathrm{d} \gamma_{\mathrm{C}, \mathrm{r}}}{\mathrm{d} \vartheta}=\operatorname{Da}_{1} \frac{1}{\mathrm{Ex}^{\mathrm{n}}}\left[\vartheta-\chi\left(2-\sigma_{\mathrm{C}}\right)\right]^{\mathrm{n}}\left(1-\frac{\chi}{\mathrm{Ex}}\right)^{\mathrm{m}} \kappa_{1}+$

$-\mathrm{Da}_{2} \frac{1}{\mathrm{Ex}^{\mathrm{p}}}\left[\vartheta-\chi\left(2-\sigma_{\mathrm{C}}\right)\right]^{\mathrm{p}}{ }_{\mathrm{C}, \mathrm{r}}{ }^{\mathrm{q}} \kappa_{2}-\mathrm{R}_{\mathrm{x}} \gamma_{\mathrm{C}, \mathrm{r}}$

with initial conditions at $\vartheta=0: \chi=0, \sigma_{C}=1$ and $\gamma_{\mathrm{C}, \mathrm{r}}=0$.

In Equations 5 to $7, \mathrm{Da}_{1}=\mathrm{k}_{1, \mathrm{R}} \mathrm{t}_{\mathrm{dos}} \mathrm{C}_{\mathrm{B} 0, \mathrm{r}}^{\mathrm{n}+\mathrm{m}-1}$ and $\mathrm{Da}_{2}=\mathrm{k}_{2, \mathrm{R}} \mathrm{t}_{\mathrm{dos}} \mathrm{C}_{\mathrm{B} 0, \mathrm{r}}^{\mathrm{p}+\mathrm{q}-1}$ are the Damköhler numbers for the main and the side reaction, equal to the ratio of the dosing time to their characteristic times; $\kappa_{1}=\exp \left[\gamma_{1}\left(1-\mathrm{T}_{\mathrm{R}} / \mathrm{T}_{\mathrm{r}}\right)\right]$ and $\kappa_{2}=\exp \left[\gamma_{2}\left(1-\mathrm{T}_{\mathrm{R}} / \mathrm{T}_{\mathrm{r}}\right)\right]$ are the dimensionless reaction rate constants; $\mathrm{R}_{\mathrm{x}}=\mathrm{t}_{\mathrm{dos}} / \tau$ is the recycle number, that is the ratio of the dosing time to the average residence time in the top reactor (Maestri and Rota, 2013 and 2015); Ex is the effective excess number, that is, the ratio of 
the initial hold-up of $\mathrm{B}$ in the reactor to the overall amount of $\mathrm{A}$ to be dosed (for a 1:1 stoichiometry).

After the supply period (that is, at $\vartheta>1$ ), Equations (5) to (7) still hold true with $\vartheta$ in the right hand side replaced by one.

For the sake of illustration, in the case of a $(1,1)$ reaction system with respect to both the reactions, the asymptotic selectivity to $\mathrm{C}, \sigma_{\mathrm{C} \infty}$, vs. the dosing time of $\mathrm{A}$ is plotted in Figures $2 \mathrm{~A}$ and $2 \mathrm{~B}$ for $E x=2$ and 1, respectively, and for different recycle number values.

It can be noticed that if a negligible dosing time were adopted (leading to a batch type reactor) the asymptotic selectivity would reach relatively high values, in particular when an excess of B is used (that is, for $\mathrm{Ex}=2$ ). This because the sudden accumulation of the whole amount of A within B leads to the reaction between the two reactants, to yield only the expected species C. However, when dealing with fast and exothermic reactions or when the uncontrolled evolution of a corrosive species could occur (as for the reaction system analyzed in this work), the sudden mixing of the two reactants in their whole amounts is not practicable because of safety constraints.

Adopting a non-negligible dosing time in a standard SBR, the coreactant accumulation in the reactor occurs within a mixture of $\mathrm{B}$ and $\mathrm{C}$, and the fed coreactant $\mathrm{A}$ can therefore unselectively react with both. For this reason, the final selectivity to $C$ quickly drops to an asymptotic lower limit, which value depends on the initially adopted excess of B. In particular, performing the reaction at a higher initial excess of the selective reactant $\mathrm{B}$, a higher final selectivity to the target product $\mathrm{C}$ is achieved, but with a lower plant productivity.

This drop of selectivity cannot be recovered by increasing the dosing time in a SBR (see the curves for $\mathrm{R}_{\mathrm{X}, \mathrm{R}}=10^{-3}$ in Figure 2, that is, almost without any recycle).

However, operating under recycle conditions of B (that is, performing the reaction in a SBRR), allows for removing the product $\mathrm{C}$ from the reactor and therefore for recovering selectivity when increasing the dosing time. In fact, if the target product $\mathrm{C}$ is generated at a higher time scale than that at which it is removed from the reactor, the extent of the undesired reaction between the fed 
reactant $\mathrm{A}$ and the target species $\mathrm{C}$ is limited and the final selectivity is enhanced, as evident from Figure 2 when the $\mathrm{R}_{\mathrm{x}}$ value increases.

Therefore the SBRR can achieve both a high selectivity and productivity.

\section{Experimental setup}

In this work the selectivity behavior of SBRR vs. SBR has been experimentally investigated through a reaction of industrial relevance in the intermediate chemical industry, that is the alkylation of ethylenediamine (EDA) with 1,2-dichloroethane (DCE) to produce triethylenetetramine (TETA):

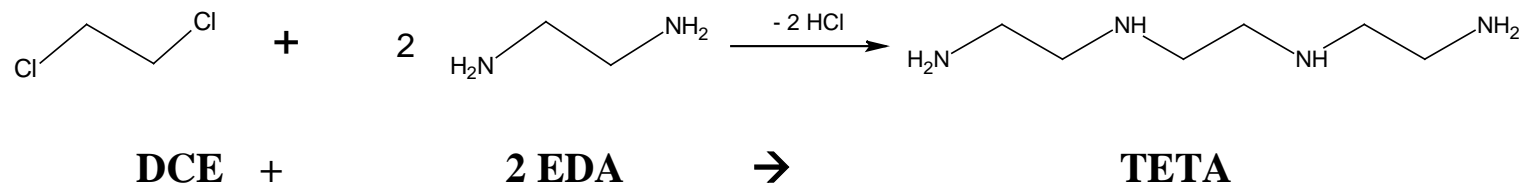

This process undergoes relevant selectivity problems since, besides the intermolecular attack of DCE to the target polyamine, more than one intramolecular attack can occur, converting ethylenediamino groups into piperazinic heterorings. Both these two classes of by-products, characterized by different DCE/EDA ratios, are often difficult to separate through conventional distillation, because of their close boiling points and the presence of multicomponent azeotropes (Hiraga et al., 1986). For these reasons, TETA is available on the market with a GC assay of the order of 60\% (Garner and Nunes, 1973; Akzo Nobel, 2018; Huntsman, 2018).

When a better TETA quality is required, more sophisticated separation techniques than conventional distillation should be adopted, making however the process uneconomical.

Taking into account that the EDA boiling point (that is, $116^{\circ} \mathrm{C}$ ) allows for distilling it at ordinary conditions, the selectivity of the DCE/EDA alkylation system can be enhanced even in a noncontinuous reaction system adopting the SBRR process configuration, that is refluxing EDA through the system and dosing DCE over a suitable time. 
Such a process solution has been experimentally tested through a laboratory plant assembled on the basis of the scheme reported in Figure 1 (Sacco, 2017). The plant consists of a top reactor and a bottom separation unit, through which the unconverted excess of EDA (used also as a solvent) is continuously recycled. Due to the relevant volatility difference between EDA and the polyamines (the main and most volatile of which is TETA), the separation unit consists of a single stage bottom reboiler. In other words, EDA and TETA are the light and heavy key components, respectively, of the separation itself, with a significant normal boiling point difference $\left(116.9^{\circ} \mathrm{C}\right.$ for EDA and $276.5^{\circ} \mathrm{C}$ for TETA).

With reference to the process scheme of Figure 1, DCE is fed through a metering pump to the top reactor, in which the liquid level is kept at a desired value through a weir. The stream leaving the reactor is continuously discharged into the bottom reboiler, where the separation between unconverted EDA and the mixture of TETA with the heavier alkylation by-products occurs. EDA vapors are then condensed and subcooled below the reaction temperature in a top exchangersubcooler, from which the condensate is finally recycled back to the reactor. Taking into account that the involved alkylation reactions are relatively fast, the DCE accumulation in the system is normally negligible. Moreover, adopting a DCE dosing time much higher than the average residence time of the reaction mass in the reactor an effective segregation between DCE and TETA can be achieved. As a consequence, the extents of the side alkylation reactions are significantly limited, even operating the reactor at a lower EDA excess, which leads to both an increased process selectivity and productivity.

When performing the SBRR reaction runs, 347mL of EDA (that is, $5.191 \mathrm{~mol}$ ), are loaded in the system and the height of the weir is adjusted in order to keep a $42 \mathrm{~mL}$ holdup in the top reactor. EDA is then heated up to reflux conditions with an initial rate of $1.15 \mathrm{~L} / \mathrm{h}$. Once a stable reflux regime is reached, the supply of $51.4 \mathrm{~g}$ of DCE (that is, $0.519 \mathrm{~mol}$ ) is started at the selected flowrate. After the whole DCE amount has been fed, a 30 minutes completion period is adopted, and the system is finally cooled down. 
The holdup reactor and the weir are then disconnected and all the mixtures are transferred into the bottom vessel, where the workup of the final product is performed (Hiraga et al., 1986; Sacco, 2017). It must be pointed out that the workup procedure to get the final product is critical for the reliability of the experimental results and must therefore be performed minimizing any physical loss of TETA.

The raw reaction mixture is in the form of hydrochlorides of the amines, arising from the reaction between the generated hydrogen chloride and the amino groups. Therefore, the aforementioned hydrochlorides must be first converted into the corresponding amines through a suitable excess of sodium hydroxide (loaded as a 50\% weight water solution), in order to recover quantitative amounts of free amines. The excess of EDA and the water generated by the reaction of sodium hydroxide with the amino hydrochlorides is then removed through atmospheric distillation. The incidental presence of traces of polyamines in the distilled mixture is checked via GC analysis. Afterwards, about $300 \mathrm{~mL}$ of $\mathrm{p}$-xylene are loaded in the vessel and the resulting mixture is kept under reflux conditions for one hour. The resulting lower viscosity solution is then filtered through a glass fiber filter at $90^{\circ} \mathrm{C}$ in order to mechanically separate the sodium chloride and the excess of sodium hydroxide (corresponding in all the performed experiments to the $30 \%$ of the theoretical alkyl halides equivalents). A second extraction of the mass remaining in the flask is then performed through further $50 \mathrm{~mL}$ of p-xylene, with which the wet cake is then washed. A clean yellowish liquid is in this way obtained after filtration and finally loaded in a Rotavapor equipment where pxylene is distilled off, leaving the final polyamine mixture, to be analyzed through GC technique.

It must be pointed out that the described filtration of sodium chloride is likely to cause significant physical losses of final product, if not adequately carried out at the laboratory scale. This because the amount of sodium chloride to be separated is relatively high compared with the expected final product (due to the relatively high molecular weight of chlorine), and the filtration itself is difficult, which limits the solid weight percentage in the final cake to $20 \%$. Therefore, the best way to proceed in this phase is estimating the cake wet fraction by subtracting from the wet cake mass the 
amount of solids, which, on the contrary, can be accurately calculated taking into account that all the initial equivalents of organic chlorine of DCE are quantitatively converted into sodium chloride. The amount of product lost in such a wet fraction can be finally estimated on the basis of the pxylene/polyamines ratio in the mother solution, which can be calculated knowing the whole pxylene amount added to the raw reaction mixture and its GC assay in terms of TETA.

Such an estimated quantity of product lost in the cake wet fraction must be finally added to that contained in the polyamines solution physically isolated after the filtration and distillation operations described above, in order to calculate an effective reaction selectivity.

\section{Results and discussion}

Two sets of experiments have been performed in which DCE has been added over different dosing times to an excess of EDA under both reflux and static conditions (that is, operating the system in the SBRR and in the SBR modes, respectively), as summarized in Table 1. More details are available in Table S.1 of the Supplementary Material section. It should first of all be noticed that the SBR runs have been carried out in very similar conditions, so that they can be regarded as a measure of the reproducibility of the experiments. The corresponding results are available in Table S.2 of the same section. According to them, the final process selectivity, defined as:

$\sigma_{\mathrm{TETA}, \infty}=\frac{\mathrm{n}_{\mathrm{TETA}}}{\mathrm{n}_{\mathrm{DCE}}} \times 100$

increases from about $50 \%$ for the SBR experiments, up to about $70 \%$ for the SBRR ones, as can be noticed from Figure $3, \mathrm{n}_{\text {TETA }}$ and $\mathrm{n}_{\mathrm{DCE}}$ being the final molar amount of TETA recovered and the fed molar amount of DCE. Coherently, a final TETA assay 10\% higher in the SBRR runs than that in the SBR experiments has been found, even adopting an effective excess number one order of magnitude lower (that is, 0.65 vs. 5.5 as average values). In particular, the TETA assay resulted to be $65-70 \%$ for the SBR experiments and $75-80 \%$ for the SBRR experiments, that is about $10 \%$ above the standard commercial grade (Akzo Nobel, 2018; Huntsman, 2018) (see Table S.2 of the 
Supplementary Material section). Therefore, adopting the SBRR process configuration, the purity of the product is intrinsically higher yet in the raw product mixture and the industrial costs to bring it on the market can be strongly reduced. It must be noticed that the results obtained for the EDA/DCE system in terms of selectivity increase moving from the SBR to the SBRR process configuration are expected to be lower than those achievable for other diamine/DCE systems, because, as previously mentioned, DCE can undergo two types of side reactions: the further alkylation of TETA leading to higher oligomers (which is characteristic of any diamine/DCE reaction system) and the intramolecular alkylation of the ethylenediamino groups by DCE, to yield relatively stable (and hence thermodynamically favored) six atoms heterocycles of the 1,4piperazinic type (which is typical of the EDA/DCE system only):
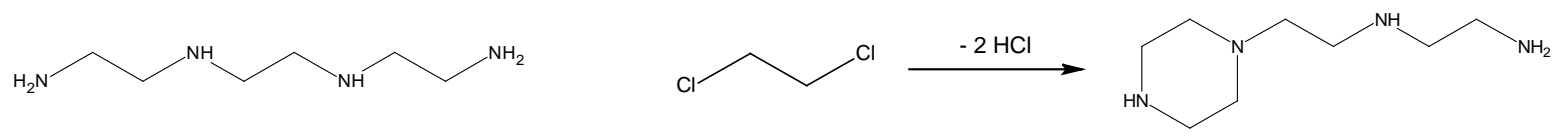

TETA

$+\quad$ DCE

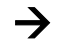

PEEDA

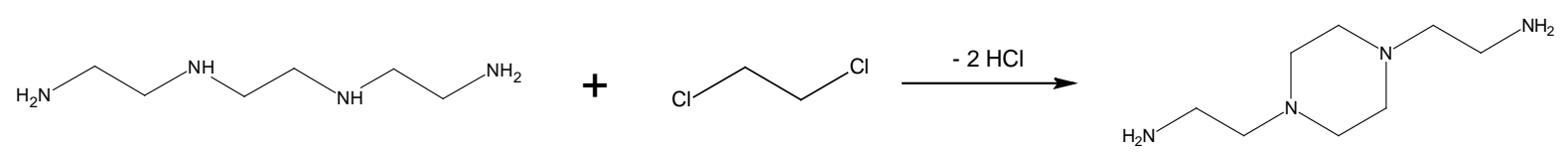

TETA

$+\quad$ DCE

$\rightarrow$

Bis-AEP

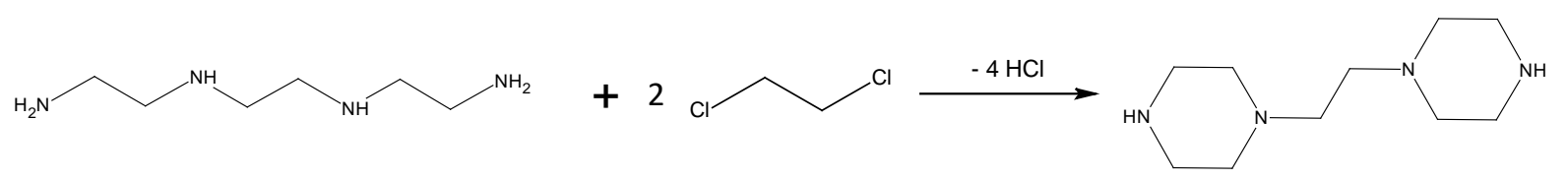

TETA +2 DCE $\rightarrow$

EBP

Such a competition of more than one undesired reaction limits the achievable selectivity gain with respect to the target polyamine. In fact, the original molecule of the target product can undergo two types of degradation paths, consisting respectively in a simple growth (towards linear or branched derivatives of higher molecular weight) and in a growth with annulation, starting with a full internal alkylation of TETA by DCE to yield ethylene-bis-1,4-piperazine (EBP): 


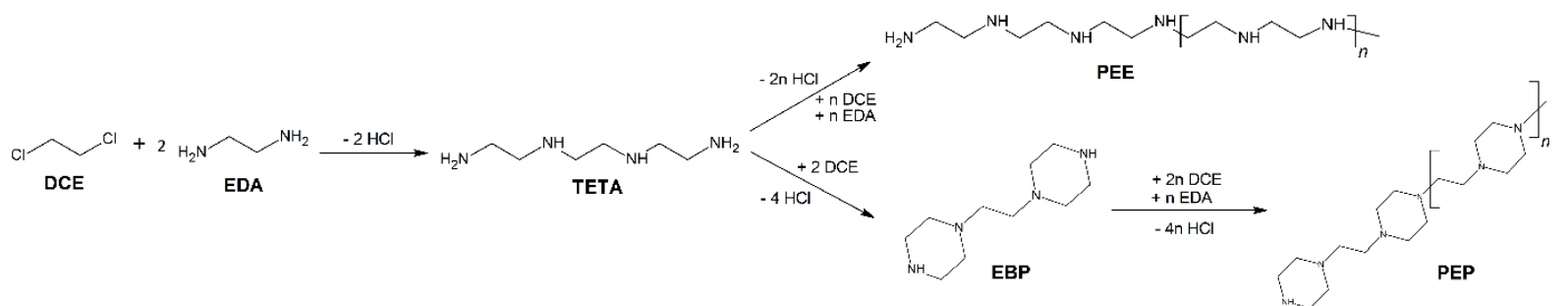

The addition to TETA and EBP (as mother species) of $n$ further monomer units of the types respectively represented in Table 2 leads to heavier derivatives, for which the DCE/EDA ratio is equal to:

$\mathrm{r}_{\mathrm{DCE} / \mathrm{EDA}, \mathrm{s}}=\frac{1+\mathrm{n}}{2+\mathrm{n}}$

$\mathrm{r}_{\mathrm{DCE} / \mathrm{EDA}, \mathrm{c}}=\frac{3+2 \mathrm{n}}{2+\mathrm{n}}$

where the labels s and c stay for simple growth or growth with cyclization.

In Figure 4, the trends of $\mathrm{r}_{\mathrm{DCE} / \mathrm{EDA}, \mathrm{s}}$ and $\mathrm{r}_{\mathrm{DCE} / \mathrm{EDA}, \mathrm{c}}$ are represented. It can be noticed that, with an initial DCE/EDA ratio equal to 1:2 for TETA and 3:2 for EBP, asymptotic ratios equal to 1:1 and 2:1 are reached for simple and cyclic growth respectively, with a maximum range of variation going from $2: 3$ to $2: 1$ at $n=1$.

When performing the reaction in a standard SBR, polyaddition reactions are more likely to occur because of the theoretically infinite residence time in the reactor. Therefore, heavier byproducts (cyclic or not) of the type represented in Table 2 should be expected.

Instead, in a SBRR operated at low residence times in the top reactor (when compared to the coreactant dosing time), the original TETA undergoes most probably single additions (either intermolecular or intramolecular), generating the different species represented in Table 2.

As a consequence, for the SBRR reaction runs a DCE/EDA molar ratio ranging from 2:3 to 1:1 is expected, whereas for the SBR experiments such a ratio can vary from 1:1 to 2:1.

More in detail, for the SBR process configuration two asymptotic situations can be figured out: the first one where the impurities consist only in not cyclic polyamines with a DCE/EDA ratio at the 
limit equal to $1: 1$, and the second one where the impurities consist of a poly-(ethylene 1,4piperazine), with an asymptotic DCE/EDA ratio equal to 3:2.

For the SBRR process configuration, instead, the two following asymptotic situations are more likely to occur, both corresponding to a single addition to TETA: the first one where a single DCE equivalent further reacts with TETA and EDA to yield penta-ethylene hexamine, with a DCE/EDA ratio equal to 2:3, and the second one according to which DCE undergoes an internal condensation with TETA, generating monopiperazinic derivatives with a DCE/EDA ratio equal to 1.

In all the aforementioned cases, the lower the DCE/EDA ratio in the impurities is, the higher the final process selectivity is, since a higher DCE amount is available to generate the target product.

On this basis, for each SBR and SBRR experimental run a range of variation for the selectivity to TETA can be estimated (from Equation 9 through some algebra), as follows:

$\sigma_{\mathrm{TETA}, \infty}=\frac{100}{1+v\left(\frac{100}{\% \text { wt TETA }}-1\right) \frac{\mathrm{M}_{\mathrm{w}, \mathrm{TETA}}}{\mathrm{M}_{\mathrm{w}, \mathrm{imp} .}}}$

where $v$ is a coefficient accounting for the molar amount of DCE per mole of impurity, $\mathrm{M}_{\mathrm{w} \text {,imp. is }}$ the molecular weight of the impurity, \% wt. TETA is the TETA weight percent in the final reaction mixture and $\mathrm{M}_{\mathrm{w}, \mathrm{TETA}}=146.24 \mathrm{~g} / \mathrm{mol}$ is the molecular weight of TETA.

This relation, for a given experimental value of \% wt. TETA in the final sample, allows for estimating an asymptotic lower and upper limit of the process selectivity by introducing the $\mathrm{M}_{\mathrm{w} \text {,imp. }}$ and $v$ values characterizing the by-products listed in Table 2.

In Figure 5, the expected selectivity ranges for the SBR and SBRR reaction runs are plotted as a function of the TETA assay for the limiting impurities summarized in Table 2 with respect to the two process configurations. It can be noticed that, as expected, the gap between the lower and upper selectivity values decreases when increasing the TETA assay.

In Figure 3 (as well as in the Supplementary Material Section) the experimental selectivity values measured in the SBR and SBRR runs are compared with the lower and upper selectivity limits 
estimated through Equation 16. It can be noticed that in all the cases the experimental selectivity resulted to be in the estimated range.

In particular, the experimental selectivity values for the SBR runs are closer to their expected upper limits than those for the SBRR runs. Such a result suggests that even with a theoretically infinite residence time in the reactor, the generation of impurities with a DCE/EDA ratio close to the top 2:1 value (corresponding to poly-ethylene-piperazinic structures of theoretically infinite length) is less realistic. In the SBRR runs, instead, a selectivity value closer to an expected average value has been achieved, hence confirming that the real impurity pattern is reasonably close in terms of DCE/EDA ratio to that of the monoaddition byproducts reported in Table 2.

Finally, as can be noticed from Figure 3, in the operating conditions investigated for the SBRR runs, a nearly constant selectivity to TETA has been found, which confirms that the corresponding recycle numbers are always above the threshold value for an effective removal of the target product from the reactor: such a general behavior of SBRRs is expected from Figure 2 at relatively high values of the $\alpha$ parameter.

\section{Conclusions}

A number of potential runaway reaction processes performed in non-continuous plants undergo selectivity drops because of the undesired reaction of one reactant with the intermediate target product.

In these cases, when a relatively high volatility gap exists between the selective reactant and the target product, a combined selectivity and productivity increase can be achieved through the SBRR process configuration, which can be easily assembled with the equipment available in any multipurpose plant (Maestri and Rota, 2013 and 2015).

In this work the selectivity behavior of the SBRR process configuration vs. a standard SBR one has been experimentally investigated through a case-study of industrial relevance, that is, the alkylation of ethylenediamine through 1,2-dichloroethane to produce triethylenetetramine. 
Adopting the SBRR process configuration a relevant increase of both the process selectivity and productivity has been achieved, generating yet in the reaction step a product of higher purity than that available on the market. 


\section{Nomenclature}

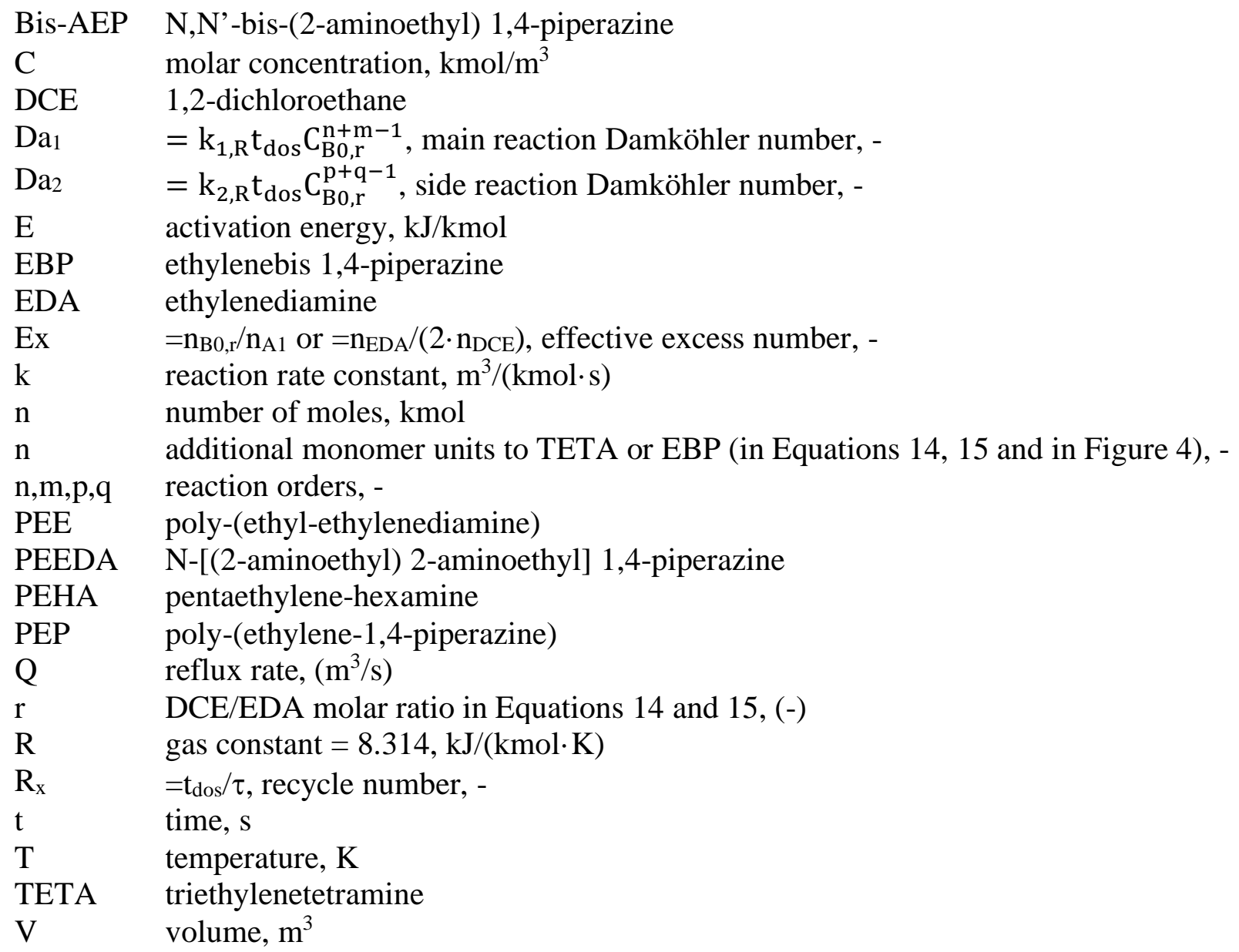

\section{Subscripts and superscripts}

$\begin{array}{ll}\text { A,B,C,D } & \text { components A, B, C and D } \\ \text { C } & \text { growth with cyclization } \\ \text { DCE } & \text { 1,2-dichloroethane } \\ \text { dos } & \text { dosing stream or dosing time } \\ \text { imp } & \text { impurity } \\ \text { r } & \text { reactor } \\ \text { R } & \text { reference } \\ \text { S } & \text { simple growth } \\ \text { TETA } & \text { triethylenetetramine } \\ \text { X } & \text { in the recycle number } \mathrm{R}_{\mathrm{x}} \\ 0 & \text { start of the semibatch period } \\ 1 & \text { main reaction or end of the dosing period } \\ 2 & \text { side reaction } \\ \infty & \text { asymptotic value in } \sigma_{\mathrm{C} \infty}\end{array}$

Greek symbols

$\alpha \quad=\mathrm{t}_{\text {dos }} / \mathrm{t}_{\mathrm{dos}, \mathrm{R},}-$ 
$\gamma \quad$ dimensionless concentration, -

$\vartheta \quad=\mathrm{t} / \mathrm{t}_{\mathrm{dos}}$, dimensionless time, -

$\kappa=\mathrm{k} / \mathrm{k}_{\mathrm{R}}$, dimensionless reaction rate constant, -

$v \quad$ DCE/byproduct molar ratio, -

$\sigma \quad=n_{\mathrm{C}} /\left(\mathrm{n}_{\mathrm{B} 0}-\mathrm{n}_{\mathrm{B}}\right)$, molar selectivity, -

$\tau \quad=\mathrm{V}_{\mathrm{r}} / \mathrm{Q}$, average residence time in the reactor, $\mathrm{s}$

$\chi \quad=\left(\mathrm{n}_{\mathrm{B} 0}-\mathrm{n}_{\mathrm{B}}\right) / \mathrm{n}_{\mathrm{A} 1}$, normalized conversion, - 


\section{$\underline{\text { References }}$}

Arpe, H. J., (2010). Industrial Organic Chemistry, fifth ed., Wiley-VCH, Weinheim.

Akzo Nobel, (2018). Environmental Product Declaration - Ethylene Amines. https://www.akzonobel.com.

Copelli, S., Derudi, M., Maestri, F., Rota, R., (2010). Safe Operating Conditions for Semibatch Processes Involving Consecutive Reactions with Autocatalytic Behaviour. Chem. Eng. Sci. 65, 5464-5470.

Dimian, A., Bildea, C., Kiss, A., (2014). Integrated Design and Simulation of Chemical Processes, Volume 13, second ed., Elsevier Science.

Garner, P. J., Nunes, C. P., (1973). Ethylene Amines. Production and Uses. Rev. Port. Quím. 15, 158-165.

Häussling, L., Neuhauser, H., Paulus, W., (1998). Preparation of Amines from Compounds having at least 3 Cyano Groups. U.S. Patent 5,750,788.

Hiraga, Y., Murakami, T., Saito, H., Fujii, O., (1986). Process for Recovering Ethylene Amines. U.S. Patent 4,582,937.

Huntsman, (2018). Ethylenamines - A Global Profile of Products and Services. https://ir.huntsman.com.

Maestri, F., Rota, R., (2013). Selectivity Problem for Fine Chemical Reactions leading to Non Volatile Products: Process Configuration and Boundary Diagrams. Chem. Eng. Sci. 90, 1-8.

Maestri, F., Rota, R., (2015). Kinetic-Free Selectivity Control of Chemical Reactions leading to Non Volatile Products. Chem. Eng. Sci. 134, 708-720.

Maestri, F., Rota, R., (2016). Kinetic-free Safe Operation of Fine Chemical Runaway Reactions: a General Criterion. Ind. Eng. Chem. Res. 55, 925-933.

Sacco, F., (2017). Development of a Semi Batch Recycle Reactor (SBRR) for the Selective Synthesis of Triethylenetetramine TETA. Master Degree Dissertation in Industrial Chemistry, Università degli Studi di Milano, Milano, IT. 
Steinbach, J., (1999). Safety Assessment for Chemical Processes, Wiley-VCH, Weinheim.

Westerterp, K. R., van Swaaij, W. P. M., Beenackers, A. A. C. M., (1984). Chemical Reactor Design and Operation, Wiley, Chichester. 
Tables

Table 1: Operating conditions of the SBR and SBRR runs, carried out at $50^{\circ} \mathrm{C}$.

\begin{tabular}{|c|c|c|}
\hline Run & Ex number & $\mathbf{R}_{\mathbf{x}}$ number \\
\hline SBR1 & 5.91 & 0 \\
\hline SBR2 & 5.43 & 0 \\
\hline SBR3 & 5.46 & 0 \\
\hline SBR4 & 5.51 & 0 \\
\hline SBR5 & 5.44 & 20 \\
\hline SBRR1 & 0.65 & 24 \\
\hline SBRR2 & 0.65 & 38 \\
\hline SBRR3 & 0.67 & 54 \\
\hline SBRR4 & 0.65 & 101 \\
\hline SBRR5 & 0.65 & 115 \\
\hline SBRR6 & 0.65 & 141 \\
\hline SBRR7 & 0.65 & 172 \\
\hline SBRR8 & 0.65 & 237 \\
\hline SBRR9 & 0.65 & 239 \\
\hline SBRR10 & 0.92 & \\
\hline
\end{tabular}


Table 2: Expected classes of TETA impurities with respect to the DCE/EDA ratio for SBR and SBRR runs.

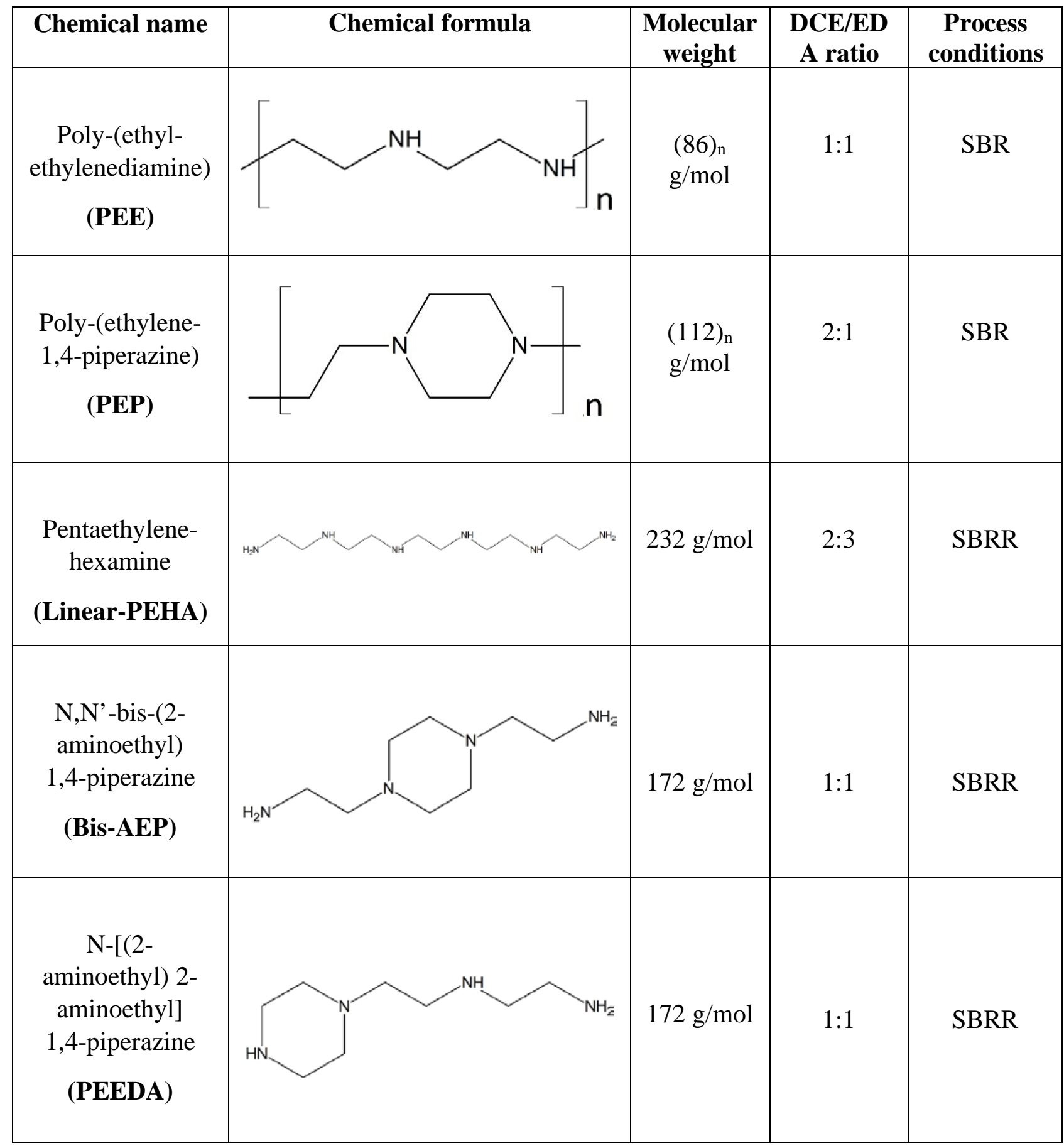




\section{Captions to the figures}

\section{Fig. 1.}

SBRR process configuration for the selective synthesis of triethylenetetramine.

\section{Fig. 2.}

SBRR process configuration. $\gamma_{1}=\gamma_{2}=30 ; \mathrm{n}=\mathrm{m}=\mathrm{p}=\mathrm{q}=1 ; \mathrm{T} / \mathrm{T}_{\mathrm{R}}=1.1 ; \mathrm{Da}_{1}=\mathrm{Da}_{2}=1$ at $\mathrm{t}_{\mathrm{dos}}=\mathrm{t}_{\mathrm{dos}, \mathrm{R}}$. Final selectivity to $C$ vs. dimensionless dosing time for $E x=2(A)$ and $E x=1(B)$.

\section{Fig. 3.}

Selective synthesis of triethylenetetramine. Experimental selectivity vs. recycle number. (o) Experimental values. (I) Estimated range of selectivity values based on the expected by-products.

\section{Fig. 4.}

DCE/EDA molar ratio in the expected byproducts vs. equivalents of further reacted EDA.

\section{Fig. 5.}

Expected selectivity ranges vs. final TETA assay for the SBR and SBRR process configurations. Comparison with the experimental results for the SBR $(\wedge)$ and SBRR (o) reaction runs. 


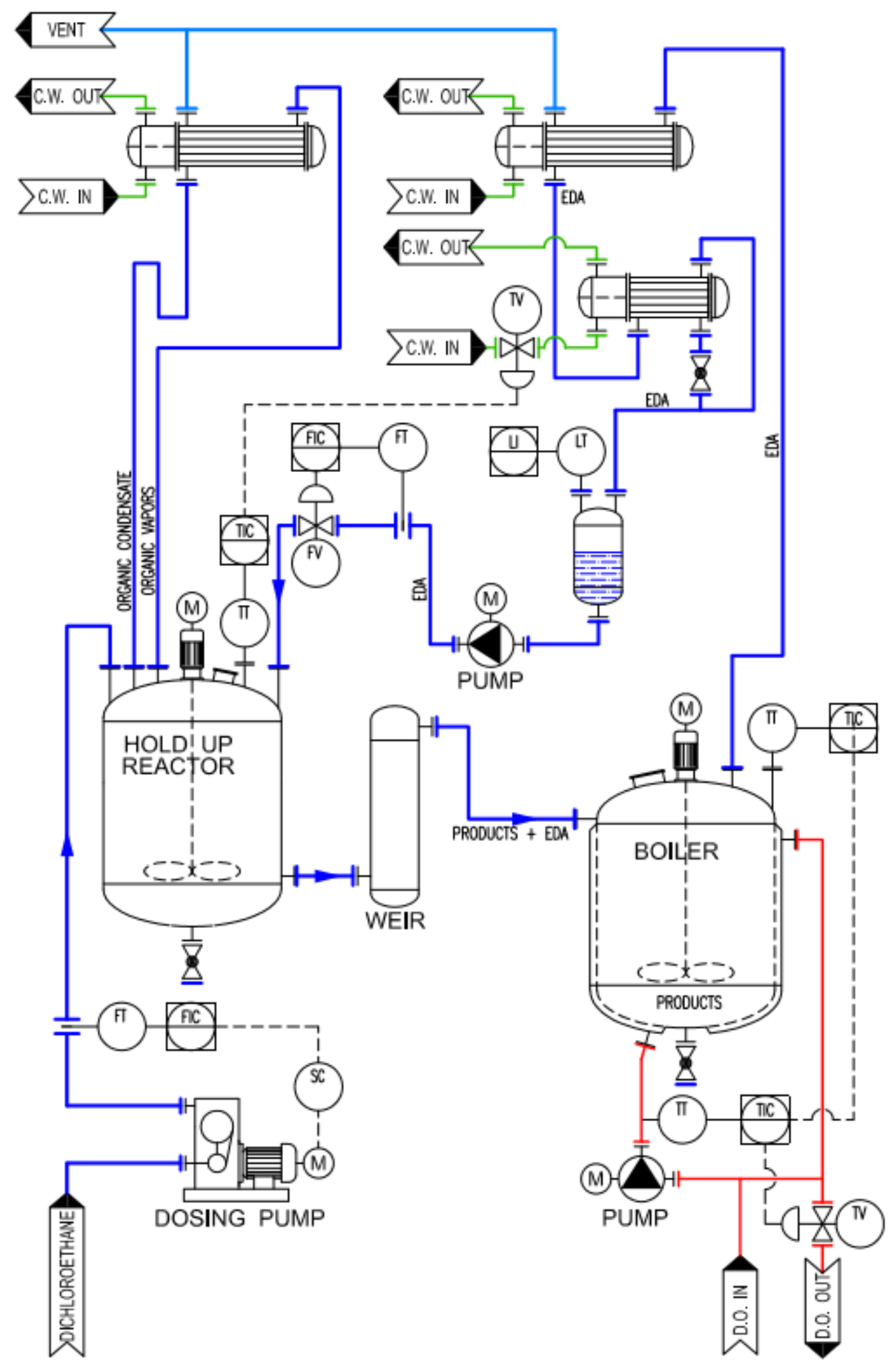

Fig.1 

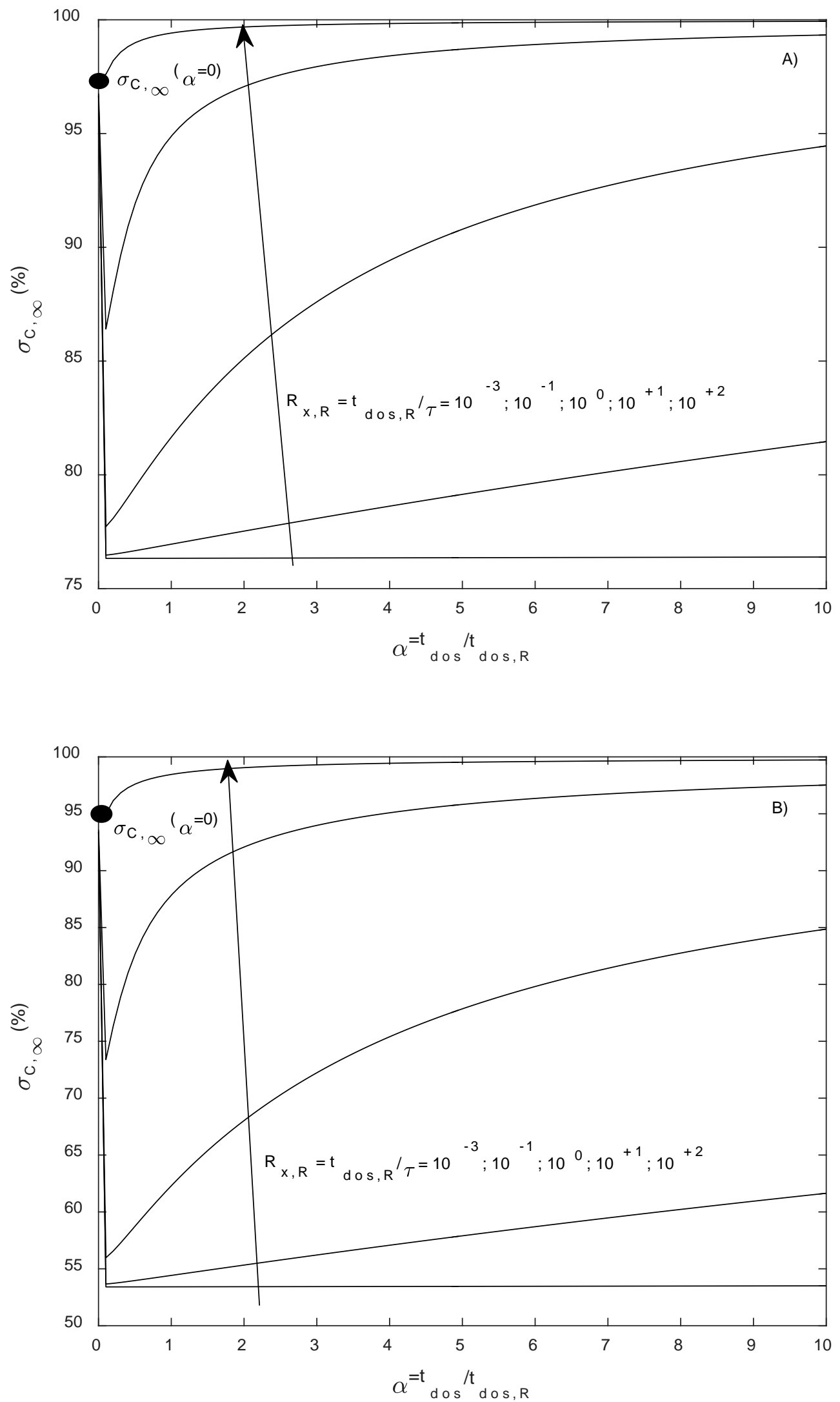

Fig. 2 


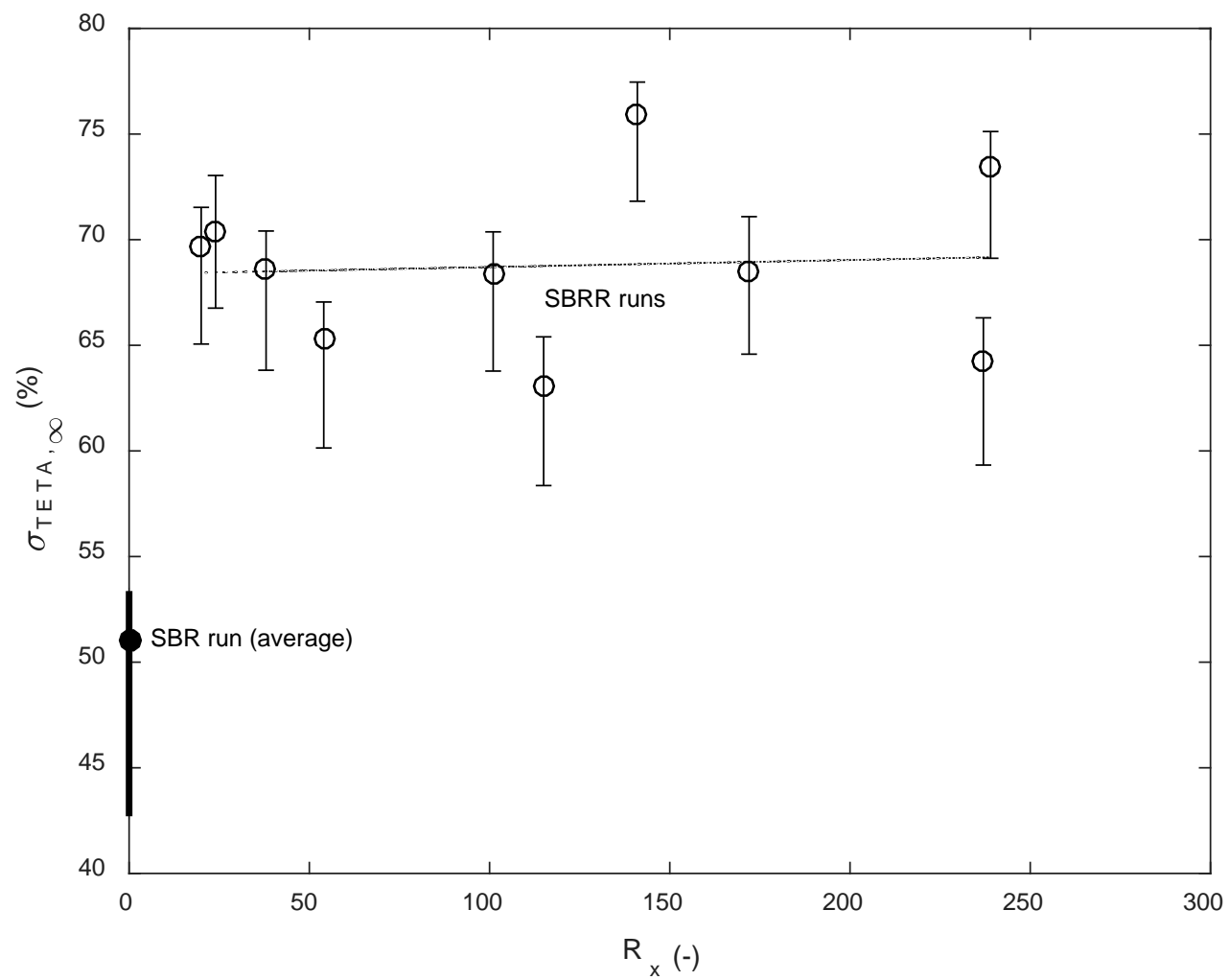

Fig. 3 


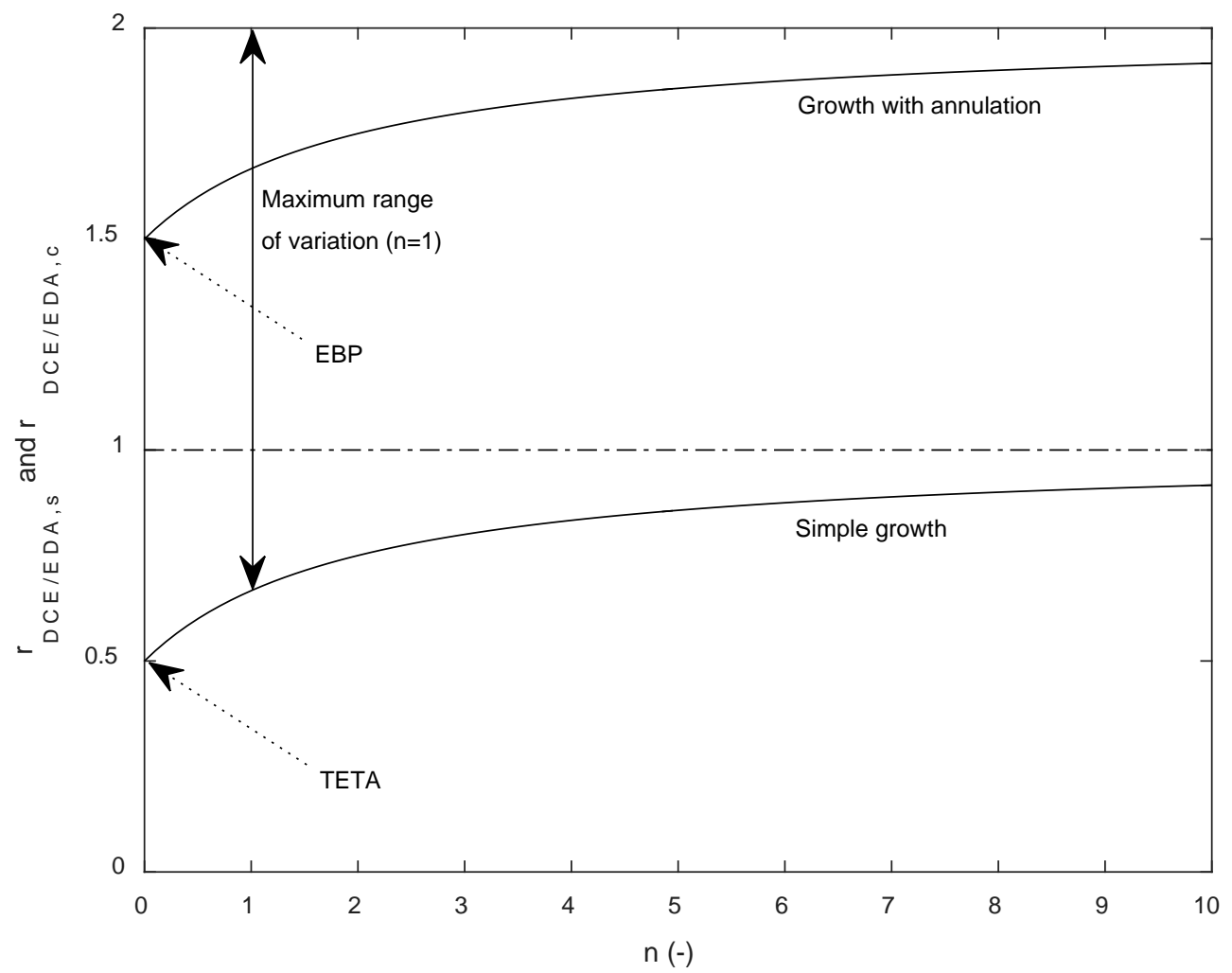

Fig. 4 


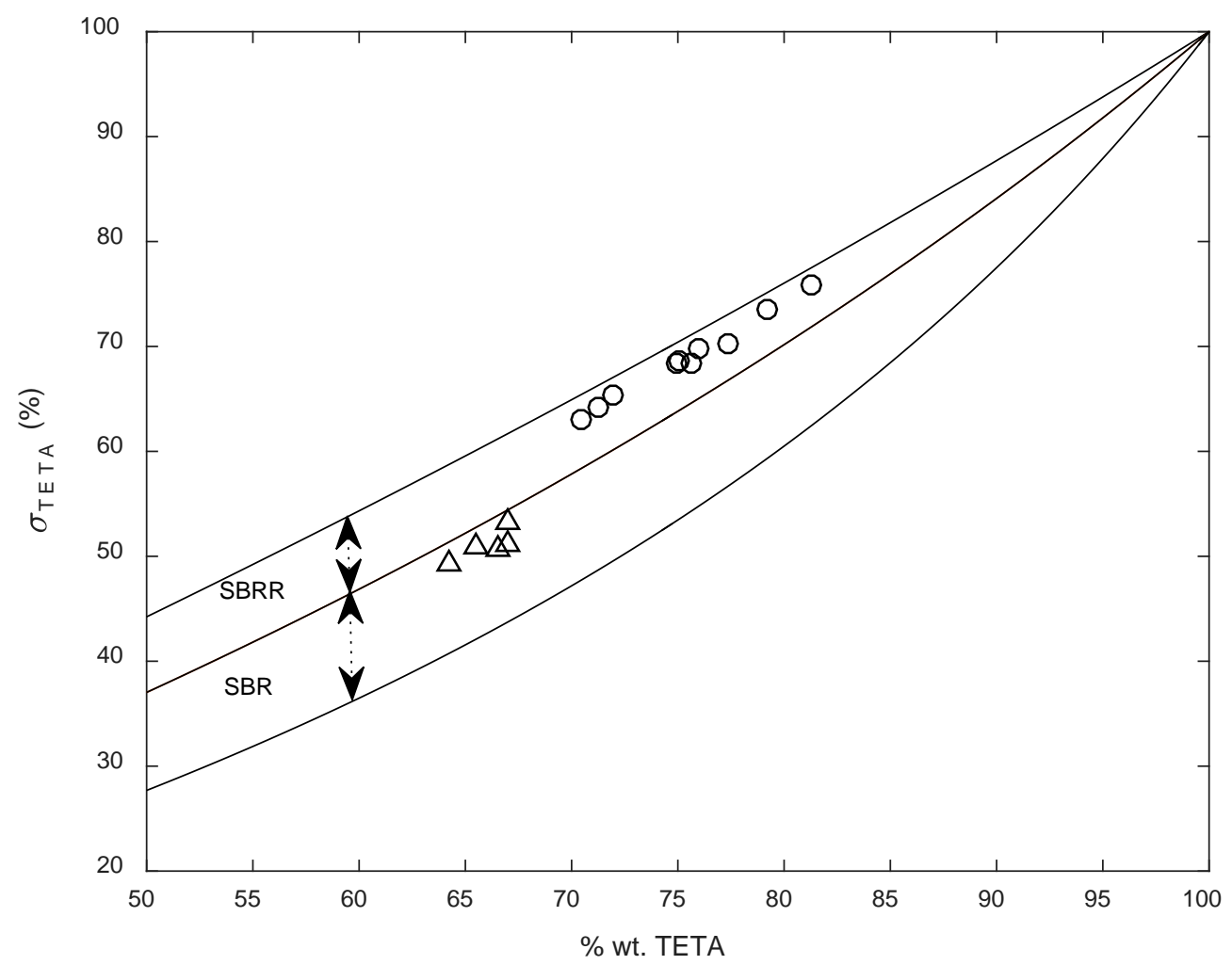

Fig. 5 